\title{
SUBHARMONIC GENERATION OF TRANSVERSE OSCILLATIONS INDUCED BY INCIDENT REGULAR WAVES
}

\author{
Gang Wang' and Jin-Hai Zheng ${ }^{2}$
}

\begin{abstract}
It is generally accepted that there are transverse oscillation, which are concentrated and confined to the backwall and decay asymptotically offshore, existed in the harbor of constant slope, however, whether these oscillations can be induced by the normally incident waves is not clear. This numerical investigation aims at providing the subharmonic generations of transverse oscillations within the harbor of a plane slope by waves normally impacting on. For the harbor of perfectly plane slopes, the subharmonic transverse oscillations are small on the mild and moderate slopes but evident on the steep slope. This instability can take place only if the incident wave amplitude exceeds a threshold value, and transverse oscillations can even grow up to a larger value than that of longitudinal oscillations. The magnitudes of transverse oscillations are approximately the same, only their growth rates are affected by the incident wave amplitude.
\end{abstract}

Keywords: Harbor Resonance; Transverse Oscillations; Instabilities; Numerical Experiments; Nonlinear Response

\section{Introduction}

Harbor oscillations are excited by the resonance due to the agreement of the eigenvalues for the free oscillations of harbor and the period of incident waves. It can cause the resonance between the moorage system, which induces many troubles such as flooding of wharves, cargo work obstacles, breaks of the mooring ropes, damage of moored vessels or infrastructures, and impaired harbor operations. Many authors have conducted research on the resonance of a harbor to oceanic waves, and a number of research papers and reports haves been devoted to addressing various aspects of this problem. Some of them have described their existence and characteristics (De Jong and Battjes, 2004; Okihiro and Guza, 1996; Wang et al., 1987). Some focused their attention on their origins, which could be wave groups (infragravity waves), atmospheric pressure disturbances, tsunamis induced by landslides or earthquakes, a shear flow and so on (De Jong and Battjes, 2004; Fabrikant, 1995; Girolamo, 1996; Kulikov et al., 1996). Much attention has also been paid to develop more accurate and more complicated models for calculating harbor oscillations with complex shapes (Losada et al., 2008; Shi et al., 2001; Woo and Liu, 2004).

Additionally several researchers addressed the analytical aspects of harbor theory. The bays and harbors under consideration have been those with simple geometry (a harbor with one basin or a basin with an entry channel) (Carrier et al., 1971; Miles, 1971) or those with complex geometry (a harbor with more than one basins or a coupled bay-river system) (Marcos et al., 2005; Mei and Ünlüata, 1976; Yu, 1996). Although these simplified analytical models developed for idealized geometries could not be applied in engineering directly, they have helped developed understandings of the rough physical mechanism, the influence of the geometric and the general features of the harbor resonance problem. Most of these theoretical studies assumed that the water depth was constant both inside and outside the harbor. The exception is the study by Marcos et al. (2005), Mei and Ünlüata (1976) and Yu (1996), which developed formulations to describe the response of harbors with variable depth. However, these solutions are implicit and need to be solved numerically. Based on the linear shallow-water approximation, Wang et al. (2011) presented an explicit formulary description of longitudinal oscillations inside a harbor with constant slope open to the sea, and investigated the influence of the depth parameters on harbor resonance. The oscillation amplitudes and the positions of nodes (antinodes) within the harbor are modified by the variable depth. Furthermore, due to refraction of the waves on variable depth, there could be several transverse oscillation modes existing in the harbor. These transverse oscillations, described as confluent hypergeometric function of the second kind, are one of a class of trapped motion concentrated and confined to the backwall and decaying asymptotically to zero far away from the backwall. However, the generation mechanism of these transverse motions was not determined; and whether they can be excited by the incident waves needs further investigations.

\footnotetext{
${ }^{1}$ State Key Laboratory of Hydrology-Water Resources and Hydraulic Engineering, Hohai University, Nanjing, 210098, China

${ }^{2}$ College of Harbour, Coastal and Offshore Engineering, Hohai University, Nanjing, 210098, China
} 
It is known that edge waves can be excited by the incident waves with a frequency equally or twice of themselves (Buchan and Pritchard, 1995; Guza and Davis, 1974). As transverse oscillations within a harbor of constant slope, together with edge waves over a sloping beach, are describe by the confluent hypergeometric functions (Edge waves are describe by Laguerre polynomials, which are the degeneration of confluent hypergeometric function of the first kind, and transverse oscillations are described by confluent hypergeometric function of the second kind), they are expected to be excited by incident waves. However, the hypothesis needs to be further confirmed, which is the motivation of our study.

During the last two decades, Boussinesq-type equations, with dramatically improved dispersion characteristics and nonlinear properties, have been solved, using finite difference methods on structured Cartesian meshes traditionally or in generalized curvilinear coordinates (Fuhrman and Madsen, 2009; Shi et al., 2001; Wei et al., 1995) and finite element method dividing the domain up into triangular or quadrilateral grids (Li et al., 1999; Losada et al., 2008). Boussinesq models have been shown to be accurate and revealing predictors of a wide range of nearshore hydrodynamic behavior, including wave propagation and shoaling, wave current interaction, wave breaking and the generation of nearshore circulation, wave-structure interaction and a range of additional topics. Furthermore, the increasing of computer power brings the modeling technique into the realm of practical calculations, especially for these discretized in curvilinear coordinates or on triangular grids, which can be well suited to handling complicated geometries and generally considered to be very robust. For these reasons, the FUNWAVE 2.0 (Fully Nonlinear Boussinesq Wave Model on Curvilinear Coordinates) model (Kirby et al., 2003), which is freely available to the public, is used in the investigation.

The description of numerical experiments concerning transverse oscillations within a harbor of constant induced by a normally incident monochromatic wave from the open sea is illustrated in section 2. The subharmonic generation of transverse oscillations is investigated in section 3 . Whether a wave train of angular frequency $2 \omega$ normally propagating into the harbor can transfer energy to small disturbances in the form of transverse oscillations characterized by angular frequency $\omega$ and these perturbations can grow up to a not been ignored amount compared with the incident wave is examined. Some results are summarized and discussed in section 3 .

\section{Description of the numerical experiments}

\subsection{Numerical model}

The FUNWAVE 2.0 model proposed and developed at the University of Delaware (Kirby et al., 2003) has been used in the present investigation. The governing equations of the model are based on the fully nonlinear Boussinesq equations derived by Wei et al. (1995). A fourth-order predictorcorrector method is used to advance in time and first order spatial derivatives is discretized to fourthorder accuracy, and Shi et al. (2001) subsequently discretized it on the staggered grid in generalized curvilinear coordinates to adapt computations to irregularly shaped shorelines. Energy dissipation due to wave breaking is modeled by introducing an eddy viscosity term into the momentum equation, with the viscosity strongly localized on the front face of the breaking waves to enable the model to simulate surf zone hydrodynamics. Wave runup on the beach is simulated using a slot technique to represent the moving shoreline and dry land. It considers a source function to generate monochromatic and random waves. Sponge layers are placed at the ends of the domain to effectively damp the energy of outgoing waves with different frequencies and directions. The capability to predict water surface elevation and velocity parameters in near-shore regions has been well validated by its developers and successors.

\subsection{Numerical simulations}

A series of numerical experiments are conducted to investigate transverse oscillations inside a harbor of constant slope induced by waves normally incident from the distant ocean. As indicated in Fig. 1, the backwall of the harbor runs in the y direction; $x=0$ is the backwall, and $\mathrm{x}$ increases offshore. The axis $\mathrm{z}$ is positive upward from the still water level. The floor of the open sea is horizontal, and the depth of a rectangular harbor is a constant slope $s=\tan \theta$. The width of the harbor is $2 b$ (from $y=-b$ to $y=b$ ), and the length is $L$. The water depth at the backwall is $h_{0}$ and $h_{1}$ at the distant ocean.

The simulations are conducted with three different beach slopes, namely the mild slope $(s=0.02)$, the moderate slope $(s=0.06)$, and the steep slope $(s=0.1)$. It is seen from Wang et al. (2011)'s result that for the specific mode $(n, m)$ of transverse oscillations, there are $n$ node lines along the backwall 
and $m$ node lines along the sidewall. The present study investigates the mode $(0,1)$, that is, there are no node lines along the backwall and one node line at the middle of the harbor running offshore. The wave amplitude of transverse oscillations decays in offshore direction, and a small finite length of the harbor may lead to the result that transverse oscillations can extend into the open sea. A much long length can certainly avoid this effect; however, the simulations of this geometry require larger calculation time. In our study, the length $\mathrm{L}$ is determined following the rule that the wave amplitude decays to the value at the entrance less than $1 \%$ of its maximum. Table 1 shows the geometry of harbors with different slopes and their resonance frequencies of mode $(0,1)$ predicted by the theory from Wang et al. (2011). The water depth at the backwall and the width keep the same for three slopes, that is, $h_{0}=0.05 \mathrm{~m}$ and $2 b=2 \mathrm{~m}$. As the harbor lengths are different for different slopes, the water depths in the open sea are also different. So it is illogical to compare transverse oscillations induced by incident waves with the same amplitude on these three slopes. A plane wave, propagating from the open sea approaches the harbor with still water as its initial surface condition at normal incidence. When such a wave impinges on a harbor with an exact plane slope, oscillations induced by it nearly uniform across the harbor. It implies that oscillations in such situation is pure longitudinal. The difference between such longitudinal oscillations induced by incident waves with the resonance frequencies and their neighboring frequencies is very small. The amplitude of incident waves for each simulation is assigned following the rule that the amplitude of oscillations at the backwall for situations without any initial perturbations is kept approximately $0.0075 \mathrm{~m}$ for all simulations by repeating the test with different incident wave amplitudes. The subharmonic transverse oscillations are investigated, and the amplitude for each situation is list in Table 1.

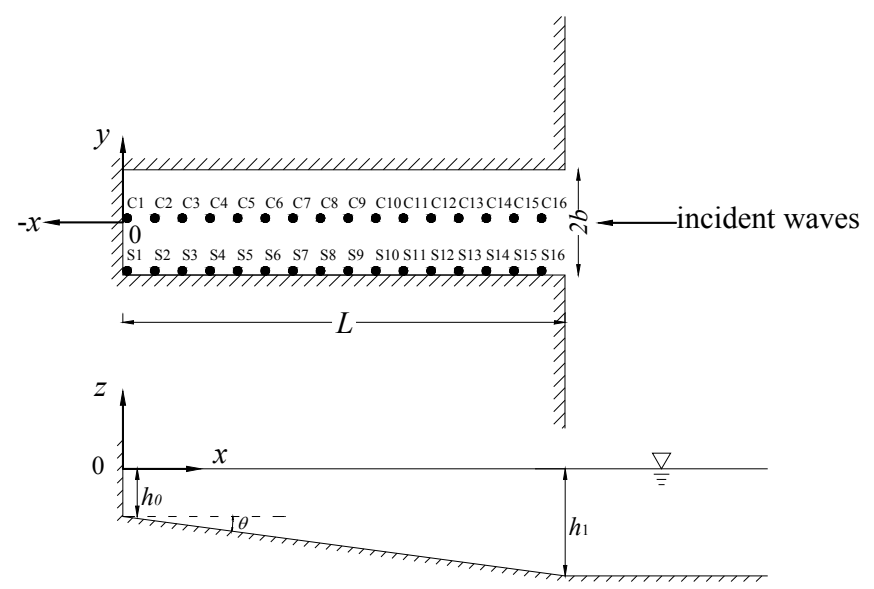

Fig. 1 Definition sketch of the harbor and coordinate systems.

\begin{tabular}{|c|c|c|c|c|c|c|c|}
\hline cases & $\begin{array}{l}\text { length } L \\
(\mathrm{~m})\end{array}$ & $\begin{array}{l}\text { width } 2 b \\
(\mathrm{~m})\end{array}$ & slope $s$ & $h_{0}(\mathrm{~m})$ & $h_{1}(\mathrm{~m})$ & $\begin{array}{c}f_{\mathrm{m}(1,0)}^{\mathrm{t}} \\
(\mathrm{Hz})\end{array}$ & $\begin{array}{c}\text { incident wave height } \\
H_{1}(\mathrm{~m})\end{array}$ \\
\hline case 1 & 5.00 & 2.0 & 0.02 & 0.05 & 0.15 & 0.212 & 0.00510 \\
\hline case 2 & 4.17 & 2.0 & 0.06 & 0.05 & 0.30 & 0.253 & 0.00485 \\
\hline case 3 & 4.00 & 2.0 & 0.10 & 0.05 & 0.45 & 0.285 & 0.00454 \\
\hline
\end{tabular}

As the numerical model used is discretized in curvilinear grids, the problem can be solved by using stretched grids in which the grid spacing is adjusted according to the water depth so that the Courant number in each element is constant in the whole domain. That is, the grid space increases gradually from the harbor to the open sea in $\mathrm{x}$ direction and keeps constant in y direction. There are approximate 40 grids in each wave length. A monochromatic wave is generated by the source function a wavelength away from the entrance. All boundaries are treated as vertical walls. Two wavelengths long sponge 
layers, one located behind the generation line and the other two installed vertically to the coastal line, are laid to absorb reflected waves and radiated waves. For all the simulated cases the number of grids fluctuates between $115 \times 121$ and $226 \times 145$, and an approximate time step of $0.01 \mathrm{~s}$ is considered. A sufficient time is considered for each simulation to assure that transverse oscillations have grown up to their maximum. For some special situations, the internal wavemaker stops working after oscillations within the harbor has reached a quasi-steady state, and the numerical model continues to simulate the damp process.

When a wave propagates into the harbor of plane slope with the still water as initial surface condition, only longitudinal oscillations are excited. However, the nature of oscillations within the harbor induced by waves with particular forcing frequencies can be changed dramatically when an apparently minor perturbation of transverse oscillations is set as the initial condition of the free surface. The excitations of subharmonic transverse oscillations are studied by setting mode $(1,0)$ transverse oscillations with the amplitude $0.0005 \mathrm{~m}$ at the backwall as the initial free surface condition within the harbor, and whether these small perturbations can get energy from the incident monochromatic wave through wave-wave interactions and grow up to a considerable magnitude is examined in the following sections. The perturbation added in our simulations is reasonable, and in practice, which can be induced by winds, landslide, topographic irregularities, etc.

Thirty-two probes are installed within the harbor, sixteen over the centre of the harbor (numbered from $\mathrm{C} 1$ to $\mathrm{C} 16$ ) and the other sixteen along the sidewall (numbered from S1 to S16), as shown in Fig. 1. As the transverse oscillation mode $(1,0)$ is investigated at present, its corresponding surface elevation $\zeta^{\mathrm{T}}$ is close to zero at the central line; however, the cross-harbor velocity $\mathrm{V}^{\mathrm{T}}$ gets its maximum here. Therefore, free surface elevations at the central probes are used to give an estimate of the longitudinal oscillation amplitude, and the cross-harbor velocities are used to estimate the transverse oscillation amplitude as there is the relation derived from the shallow-water equations:

$$
\zeta^{\mathrm{T}}=\mathrm{i} \frac{\omega}{g k_{1}} V^{\mathrm{T}}
$$

where $\mathbf{i}$ is the imaginary unit and indicates a difference phase $\pi$ here, $g$ is the acceleration of gravity, $\omega$ is the angular frequency of transverse oscillation, and $k_{1}$ is the wave number, which is $\pi / 2$ in present study. The difference in amplitudes recorded by the two probes located on the same phase lines of the longitudinal-wave field may be used to estimate the amplitudes of transverse oscillations if the phase between longitudinal and transverse oscillations remains constant. However, the phase is generally varied especially for strong nonlinear interactions (Guza and Davis, 1974). As the transverse oscillations will grow up to a rather large magnitude for the resonance situation and the nonlinear interactions between longitudinal and transverse oscillations are strong. So equation (1) is preferred in our study, and following results of transverse oscillations are all obtained by this method if they are not specially emphasized. (more detailed comparisons of the two methods are described in the following section).

\section{Subharmonic Generations}

Series of simulations are conducted with plane slopes $s=0.02,0.06$ and 0.10 to try to generate transverse oscillations within these harbors by a subharmonic interaction mechanism. Numerical points of the neutral stability and the margin of modulation for transverse mode $(1,0)$ for three cases are shown in Fig. 2. For case 1 (the mild slope) and case 2 (the moderate slope), oscillations within the harbor are mainly longitudinal, and the minor perturbation of transverse oscillations diminishes slowly and disappears completely at last. Furthermore, attempts, including simulating a wider range of neighboring frequencies and larger amplitudes, to generate evident transverse oscillations have been proved unsuccessful. As with the harbor of the steep slope (case 3), transverse oscillations appear spontaneously when the incident wave frequency is near twice the eigenfrequency of mode $(1,0)$. The numerical result of the maximum amplified frequency is slightly below the theory (its relative error is less than 2.2\%), and this decrease may be deduced to the finite-amplitude effect (Fultz, 1962).

Fig. 3 shows the time history of the surface elevation together with its wavelet amplitude spectrum for case 3 with the incident frequency $f=0.55788 \mathrm{~Hz}$ at the corner (the probe $\mathrm{S} 1$ ), where transverse oscillations and longitudinal oscillations are superposed. It is difficult to distinguish these oscillation components just from the surface elevation. However, as the energy is spread over time and frequency domains in wavelet spectrum, it is easy to recognize them as the frequency of transverse oscillations is half that of longitudinal oscillations. The wave energy is concentrated at the incident frequency when the motion appears within the harbor, and subharmonic energy corresponding to the transverse 
perturbation is very small. However, these transverse oscillations grow up slowly, and get a quasisteady state after $1500 \mathrm{~s}$. The magnitude of transverse oscillations is much larger than that of longitudinal oscillations at this stage. Indeed, the oscillations, which is the superposition of transverse oscillations and longitudinal oscillations, are so large that they have been broken in reality. The effect of breaking on the transverse oscillation generation is not investigated in present study, which is designed to be further studied with laboratory experiments. However, the phenomenon that transverse oscillations can grow up to the magnitude much bigger than that of longitudinal oscillation is credible. In addition to transverse oscillations $\left(f^{\mathrm{T}}=0.27894 \mathrm{~Hz}\right)$ and the fundamental harmonic longitudinal oscillations $\left(f^{\mathrm{L}}=0.55788 \mathrm{~Hz}\right)$, the energy, which belongs to their sum frequency $\left(f=f^{\mathrm{T}}+f^{\mathrm{L}}=\right.$ $0.83682 \mathrm{~Hz}$ ), is also present. The source function is stopped after $2000 \mathrm{~s}$, and the model continues to simulate the damp process. The energy of the fundamental longitudinal oscillation and their sun frequency with transverse oscillation diminishes quickly, and the transverse oscillations persist until the end of the simulation, which implies that the damp process of these transverse oscillations is a rather long time in practice.
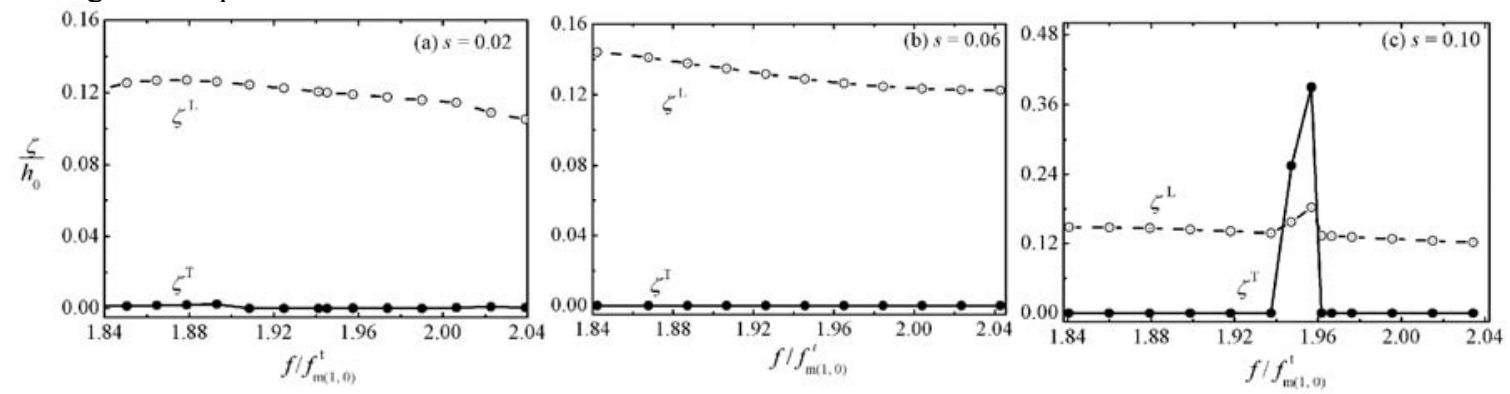

Fig. 2 Maximum wave amplitudes at the backwall as a function of the incident wave frequency for subharmonic generations of transverse oscillations. $-\circ-$ refers to longitudinal oscillation amplitude $\zeta^{\mathrm{L}}$, and $-\bullet-$ refers to transverse oscillation amplitude $\zeta^{\top}$; Notice the resonance frequencies corresponding to mode $(1,0) f_{m(1,0)}^{t}$ for three cases are different (see Table 1).

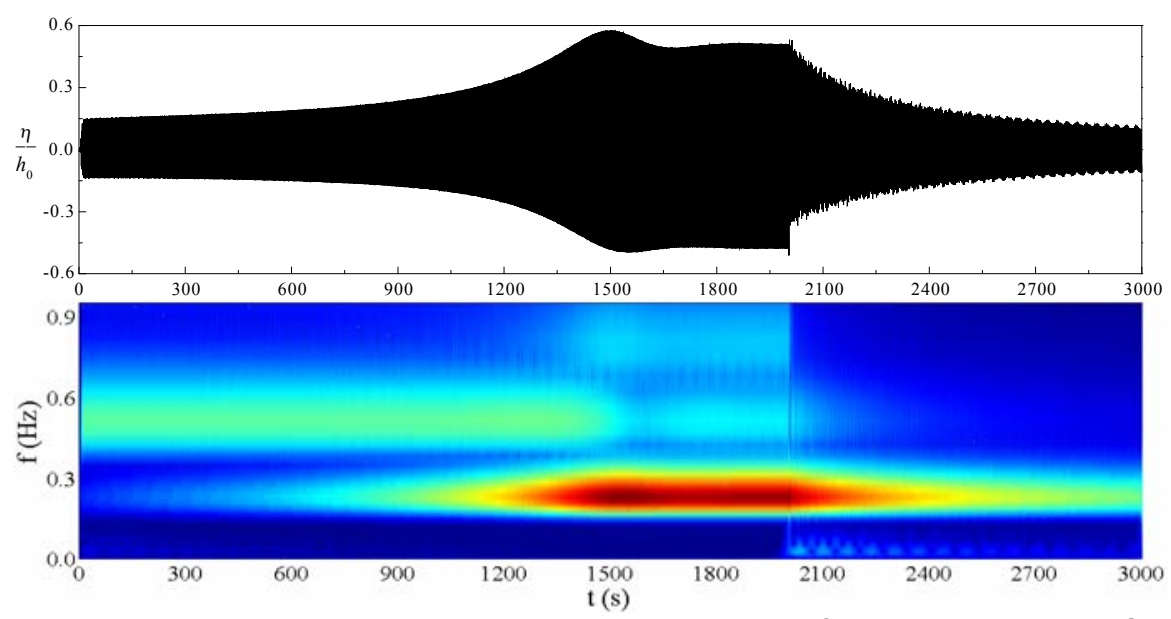

Fig. 3 Surface elevations and corresponding wavelet amplitude spectrum for case 3 at the probe $\mathbf{S 1}$ with the incident frequency $f=0.55788 \mathrm{~Hz}$, which is the maximum amplified subharmonic frequency.

Fig. 4 compares the amplitude variation of transverse oscillations and longitudinal oscillations along the sidewall. As transverse oscillations are mainly trapped near the backwall, where the depth is relatively shallow, and the theory based on a linear, weakly dispersive, Boussinesq-type equation can well describe them. Slight differences between the numerical results and the theory on longitudinal oscillations, including both amplitude and phase, can be seen from the plot. As these longitudinal oscillations distribute more energy in the region far away from the backwall, where the water is relative deep, the shallow water assumption, the analytic solution is based on, is not strictly satisfied. The snapshots of the free surface at $t=1800 \mathrm{~s}$ is shown in Fig. 5. The whole free surface in the harbor moves up and down, showing strong behavior of transverse motions.

Oscillations within the harbor of slope $s=0.1$ induced by an incident monochromatic wave with different amplitude but the same resonance frequency are simulated (see Fig. 6). For small incident waves, oscillations within the harbor are mainly longitudinal, and minor transverse perturbations decay 
to a certain even small magnitude and then keep constant. However, when the incident wave exceeds the certain magnitude, the transverse oscillations will grow up and get a steady state with amplitude larger than that of longitudinal oscillations. Although the magnitudes of transverse oscillations induced by the wave with amplitude exceeded the threshold value are approximately the same, their growth rates are different. For a incoming wave with large amplitude, transverse oscillations grow up quickly and the total oscillations within the harbor get a steady state in a 'short' time (such as the situation $H_{\mathrm{I}}$ / $h_{0}=0.12$ in our study, the time for transverse oscillations getting a steady state is about 1000 s, which is not real short compared with the period ); for the incident wave with amplitude slight larger than the threshold, the growth rate for transverse oscillations is very small (such as the situation $H_{\mathrm{I}} / h_{0}=0.08$ in our study, the response time for transverse oscillations to achieve a steady state is about 3000s).

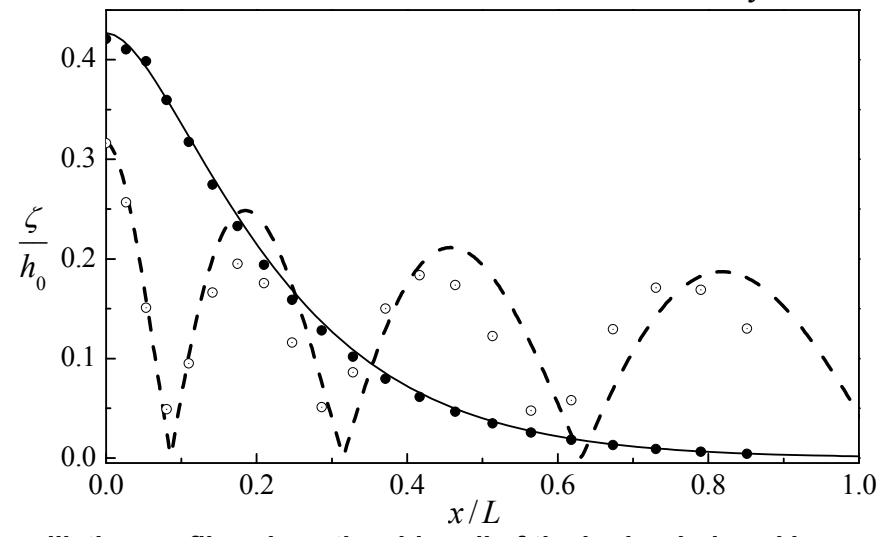

Fig. 4 Comparisons of oscillation profiles along the sidewall of the harbor induced by a monochromatic wave with frequency $f=0.55788 \mathrm{~Hz}$ and wave height $H_{1}=0.00454 \mathrm{~m}$ : $\bullet$, transverse oscillations with frequency $f^{\top}=$ $0.27894 \mathrm{~Hz} ; \circ$, longitudinal oscillations with frequency $\mathrm{f}=0.55788 \mathrm{~Hz} ;-$, theory for transverse oscillations; ----, theory for longitudinal oscillations.

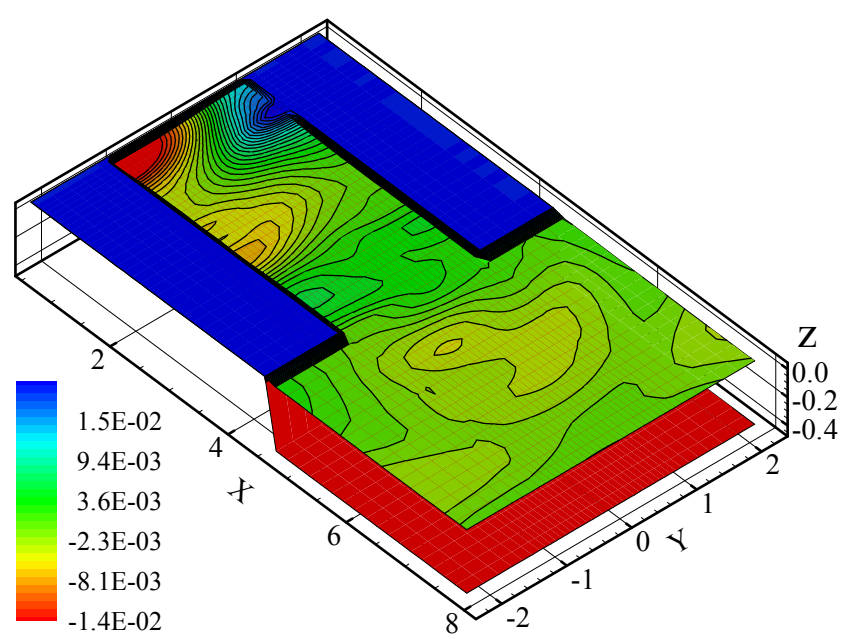

Fig. 5 Snapshot of free-surface elevation from FUNWAVE modeling results at $t=1800$ s for Case 3 , i.e., the longitudinal bottom slope $=0.1$ with incident wave frequency, $f_{1}=0.55788 \mathrm{~Hz}$.

As mentioned before, transverse oscillations can be estimated by the difference in time histories of free surface elevation recorded by the two probes located on the same phase lines of the longitudinalwave field or directly using Eq. (1). Fig. 7 compares the results estimated by the two methods for the harbor of slope $\mathrm{s}=0.10$ with a monochromatic wave of frequency $f=0.55788 \mathrm{~Hz}$ and wave height $\mathrm{H}=$ $0.00454 \mathrm{~m}$. The free surface elevation estimated by (1) is regular and symmetry, and the energy is concentrated at the eigenfrequency $f^{\mathrm{T}}=0.27894 \mathrm{~Hz}$. However, the time series obtained by the difference between two probes are irregular and asymmetric, and the energy level at the second and third harmonics is also emerged. It is known that transverse oscillations are resonance waves; the energy should be concentrated at the eigenfrequency, and it is unreasonable that there is evident energy corresponding to superharmonics. This is the reason why we abandon the method with two probes on the same phase line, and use Eq. (1) directly. 


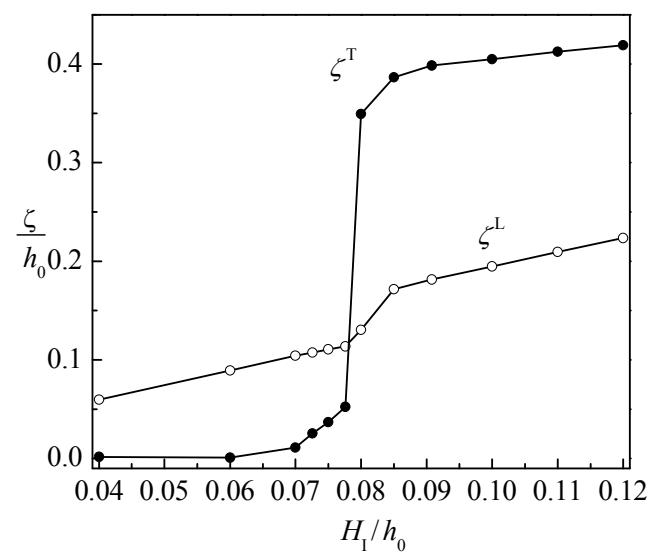

Fig. 6 Maximum amplitudes at the backwall within the harbor of slope $s=0.10$ for various incident heights but constant frequency $f=0.55788 \mathrm{~Hz}$ : $-\bullet-$, transverse oscillations $\left(f^{\top}=0.27894 \mathrm{~Hz}\right) ;-\circ-$, longitudinal oscillations $\left(f^{\mathrm{L}}=0.55788 \mathrm{~Hz}\right)$.

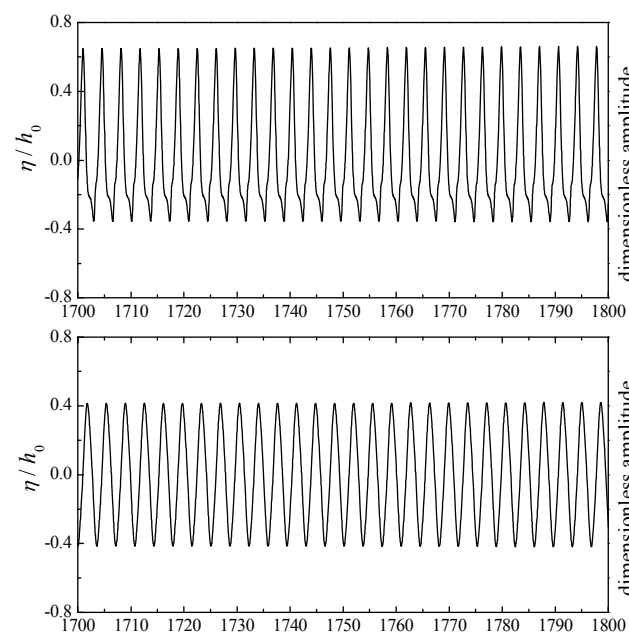

$t(\mathrm{~s})$
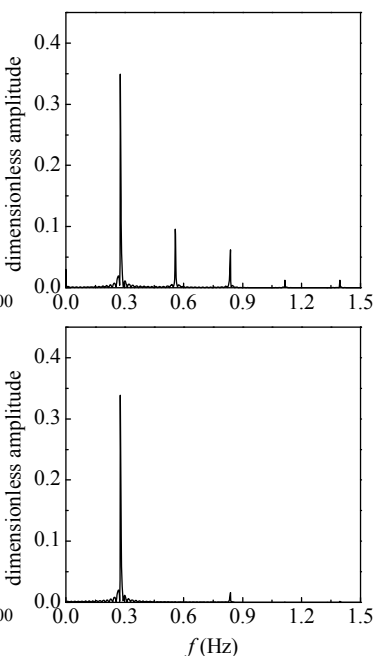

Fig. 7 Free surface elevation and amplitude spectrum corresponding to transverse oscillations estimated by the difference in time history recorded by probes $\mathrm{S} 1$ and $\mathrm{C} 1$ (upper panel) and using Eq. (1) by the crossharbor velocity $V^{\top}$ at probe $C_{1}$ (down panel) for case 3 with the incident frequency $f=0.55788 \mathrm{~Hz}$ and wave height $H_{1}=0.00454 \mathrm{~m}$.

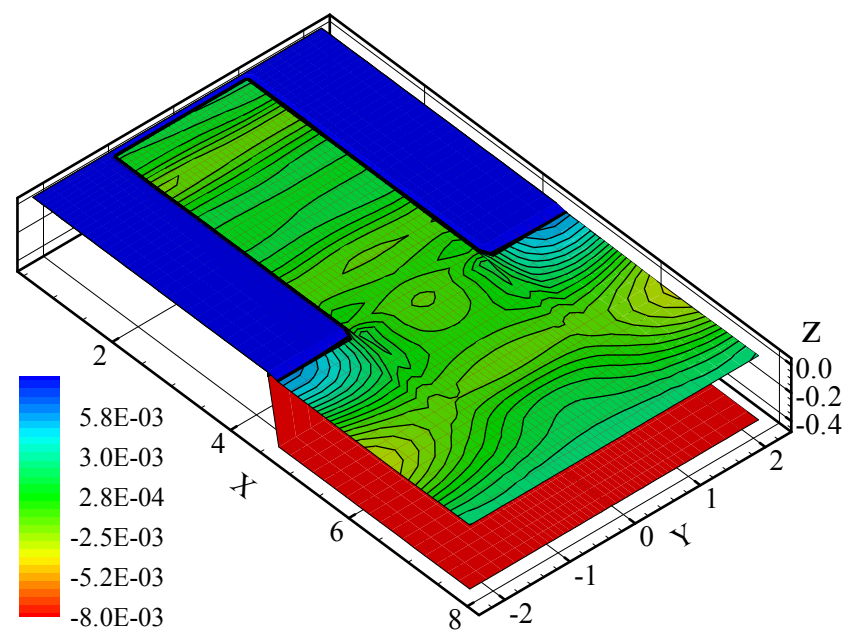

Fig. 8 Snapshot of free-surface elevation from linearly modeling results at $t=1800$ s for Case 3 , i.e., the longitudinal bottom slope $=0.1$ with incident wave frequency, $f_{i}=0.55788 \mathrm{~Hz}$.

To verify that transverse oscillation resonance is indeed caused by nonlinear process, simulations are also conducted with FUNWAVE 2.0 by flagging out the nonlinear terms. The same minor 
perturbations for providing the initial negligible small transverse oscillations remain the same. Under this situation, oscillations within the three harbor configurations are mainly longitudinal, and the provided minor perturbation of transverse oscillations does not grow up as they cannot get energy from the longitudinal waves in the linear system simulations (Fig. 8). This proves once again that 1 subharmonic generation of transverse oscillations is a nonlinear process and the transverse perturbation grows up by getting energy through nonlinear interaction.

\section{Conclusions}

The numerical experiments are carried out to investigate the mechanism by which transverse oscillations may be generated within the harbor of constant slope by waves normally incident impacting on the harbor from the open sea. As the geometry and bathymetry can be set precisely, and a clean monochromatic wave is generated by the source function, there are no inherent perturbations in simulations. So a minor perturbation of transverse oscillations is set as the initial condition of the free surface within the harbor, and the stability of a normally-incident-wave field to transverse-oscillation perturbations is investigated. The subharmonic generation, in which manner transverse oscillations generated have half the frequency of the primary wave train, is small in the harbor of the mild slope and the moderate slope but evident in the harbor of the steep slope. This instability can take place only if the incident wave amplitude exceeds a threshold value, and transverse oscillations can even grow up to a larger value than that of longitudinal oscillations. The magnitudes of transverse oscillations are approximately the same, only their growth rates are affected by the incident wave amplitude. The transverse-oscillation instability induced by a considerably larger wave reaches a steady state quickly, while it needs rather long time for the incident wave with small amplitude.

\section{Acknowledgments}

This research was supported by China Postdoctoral Science Foundation funded project (2012M511671), the Fundamental Research Funds for the Central Universities (2012B01214) and Jiangsu Planned Projects for Postdoctoral Research Funds (1102071C).

\section{References}

Buchan, S.J. and Pritchard, W.G., 1995. Experimental observations of edge waves. Journal of Fluid Mechanics, 288: 1-35.

Carrier, G.F., Shaw, R.P. and Miyata, M., 1971. The Response of Narrow-Mouthed Harbors in a Straight Coastline to Periodic Incident Waves. Journal of Applied Mechanics, 38(2): 335-344.

De Jong, M.P.C. and Battjes, J.A., 2004. Seiche characteristics of Rotterdam harbour. Coastal Engineering, 51(5-6): 373-386.

Fabrikant, A.L., 1995. Harbour oscillations generated by shear flow. Journal of Fluid Mechanics, 282: 203-217.

Fuhrman, D.R. and Madsen, P.A., 2009. Tsunami generation, propagation, and run-up with a highorder Boussinesq model. Coastal Engineering, 56(7): 747-758.

Fultz, D., 1962. An experimental note on finite-amplitude standing gravity waves. Journal of Fluid Mechanics, 13(02): 193-212.

Girolamo, P.D., 1996. An experiment on harbour resonance induced by incident regular waves and irregular short waves. Coastal Engineering, 27(1-2): 47-66.

Guza, R.T. and Davis, R.E., 1974. Excitation of Edge Waves by Waves Incident on a Beach. Journal of Geophysical Research, 79(9): 1285-1291.

Kirby, J.T., Wen, L. and Shi, F., 2003. FUNWAVE 2.0 Fully Nonlinear Boussinesq Wave Model On Curvilinear Coordinates. Technical Report No. CACR-03-XX. Center for Applied Coastal Research, Department of Civil Engineering, University of Delaware, Newark.

Kulikov, E.A., Rabinovich, A.B., Thomson, R.E. and Bornhold, B.D., 1996. The landslide tsunami of November 3, 1994, Skagway Harbor, Alaska. Journal of Geophysical Research-Oceans, 101(C3): 6609-6615.

Li, Y.S., Liu, S.X., Yu, Y.X. and Lai, G.Z., 1999. Numerical modeling of Boussinesq equations by finite element method. Coastal Engineering, 37(2): 97-122.

Losada, I.J., Gonzalez-Ondina, J.M., Diaz-Hernandez, G. and Gonzalez, E.M., 2008. Numerical modeling of nonlinear resonance of semi-enclosed water bodies: Description and experimental validation. Coastal Engineering, 55(1): 21-34.

Marcos, M., Monserrat, S., Medina, R. and Lomonaco, P., 2005. Response of a harbor with two connected basins to incoming long waves. Applied Ocean Research, 27(4-5): 209-215. 
Mattioli, F., 1978. Wave-induced oscillations in harbours of variable depth. Computers \& Fluids, 6(3): 161-172.

Mei, C.C. and Ünlüata, Ü., 1976. Resonant scattering by a harbor with two coupled basins. Journal of Engineering Mathematics, 10(4): 333-353.

Miles, J.W., 1971. Resonant response of harbours: an equivalent-circuit analysis. Journal of Fluid Mechanics, 46(02): 241-265.

Okihiro, M. and Guza, R.T., 1996. Observations of seiche forcing and amplification in three small harbors. Journal of Waterway Port Coastal and Ocean Engineering-ASCE, 122(5): 232-238.

Raichlen, F. and Naheer, E., 1976. Wave induced oscillations of harbors with variable depth, Proceedings $15^{\text {th }}$ International Conference on Coastal Engineering, Honolulu, pp. 3536-3556.

Shi, F.Y., Dalrymple, R.A., Kirby, J.T., Chen, Q. and Kennedy, A., 2001. A fully nonlinear Boussinesq model in generalized curvilinear coordinates. Coastal Engineering, 42(4): 337-358.

Wang, G., Dong, G., Perlin, M., Ma, X. and Ma, Y., 2011. An analytic investigation of oscillations within a harbor of constant slope. Ocean Engineering, 38: 479-486.

Wang, X., Li, K., Yu, Z. and Wu, J., 1987. Statistical Characteristics of Seiches in Longkou Harbour. Journal of Physical Oceanography, 17(7): 1063-1065.

Wei, G., Kirby, J.T., Grilli, S.T. and Subramanya, R., 1995. A Fully Nonlinear Boussinesq Model for Surface-Waves .1. Highly Nonlinear Unsteady Waves. Journal of Fluid Mechanics, 294: 71-92.

Woo, S.B. and Liu, P.L.F., 2004. Finite-element model for modified Boussinesq equations. II: Applications to nonlinear harbor oscillations. Journal of Waterway Port Coastal and Ocean Engineering-ASCE, 130(1): 17-28.

Yu, X.P., 1996. Oscillations in a coupled bay-river system .1. Analytic solution. Coastal Engineering, 28(1-4): 147-164.

Zelt, J.A. and Raichlen, F., 1990. A Lagrangian Model for Wave-Induced Harbor Oscillations. Journal of Fluid Mechanics, 213: 203-225. 